\title{
Research on the Arranging and Patchwork Design for Storage Tank Bottom Based on Sinovation
}

\author{
Yang Junru $^{1, *}$, Song Chuanjuan ${ }^{1}$, Zhang Nannan $^{2}$, Zhang Daozhong $^{2}$ and Xu Ning ${ }^{2}$ \\ ${ }^{I}$ College of Mechanical and Electronic Engineering, Shandong University of Science and Technology, Qingdao, \\ 266590, China; ${ }^{2}$ Shandong Hoteam Software Co., LTD., Ji'nan, 250101, China
}

\begin{abstract}
In the paper, to solve the patchwork problem of large chemical storage tank bottom, theoretical researches on arranging special-shaped parts are carried out. The heuristic layout algorithm with dynamic decision layout order and based on graphics position strategy is proposed. According to the algorithm, basing on Sinovation platform, using the object-oriented programming language $\mathrm{C}++$ and Visual Studio tools, the arranging and patchwork system is designed and developed. And the application researches are performed with the system. The results show that, comparing with the rectangular envelopment algorithm, the plate utilization ratio is increased with the patchwork algorithm as proposed in the paper.
\end{abstract}

Keywords: Arranging and patchwork system, graphics position strategy, large storage tank bottom.

\section{INTRODUCTION}

During the welding process of the petroleum chemical storage tank bottom, in order to save steel material, construction units should use the computer drawing software to optimize the design of arranging and patchwork within the specifications and design requirements, to reach lower costs, to make full use of the steel material, and to improve the production efficiency. Using a computer application program instead of manual programming is an effective method to solve the design problem of arranging and patchwork of large chemical storage tank bottom.

Up to now, there have been some references about the mature arranging theories. Liu Delian et al. [1] developed a set of arranging application program of cylindrical storage tank wall and bottom using VB language. The program contained many plate arrangements, including the butt-joint or lap and the arrangement of center plates, whether to use the edge plates and the number, specifications and the symmetry type of the edge plates, and so on. Xu Buyun et al. [2] used AutoCAD to design the arranging system for the steel vertical storage tank with six sub-systems: the vertical storage tank bottom, the vertical storage tank wall, the vertical storage tank roof, plate arrangement with no damage to the tank wall weld line, the rack layout drawings, and the tool and fixture layout drawings. Wang Dingkui et al. [3], using the mechanics formulas for the stability of thin rectangular plates, compared the critical width of two arranging welding of storage tank bottom, drew a conclusion that the arranging welding critical width for T-shape plate was smaller than that for the strip plate, and put forward to choose the strip plate first.

*Address correspondence to this author at the 579 Qianwangang Road Economic \& Technical Development Zone, Qingdao Shandong Province, China. Postcard: 266590; Tel: 0532-8068113;

E-mail: jryangzhang@163.com
The cutting stock problem after storage tank arranging, which is also called patchwork design module, belongs to the special-shaped piece layout problem that is a class of NPC problem with the highest computational complexity and can be solved with arranging [4]. Arranging can be divided into rectangular packing and irregular nesting [5]. The rectangle pieces with simple shapes have been widely used. The researches on rectangle pieces can solve not only rectangle packing problem directly, but also irregular part nesting problem by transforming two-dimension irregular pieces into rectangular parts with rectangular envelopment algorithm. Therefore, many scholars have been researched on rectangular piece packing problems for many years, and they also put forward many algorithms to optimize the packing ways [612], such as the BL algorithm based on the lower-left-most principle (lower left corner priority algorithm), BLF (bottom-left-fit) algorithm, down the step algorithm, similar graphics composition algorithm, dynamic programming algorithm, horizontal line algorithm, the lowest outline with best match algorithm, and so on.

Li Lina et al. [13], made some researches on the patchwork program excluding the deep explored work on the special shape piece packing theories. The special shape piece packing can be broadly divided into two categories: the rectangular approximation algorithm based on the regular part packing process and the direct treatment to the irregular pieces. The rectangular envelopment algorithm was used in reference [14]. The envelope rectangle of the polygon is a rectangle including all the points and contacting with all the sides of the polygon. The algorithm is suitable to the layout for the part with approximate rectangle polygon. As the envelope rectangle is an approximate graphic, when its area has a big difference with a complex polygon, the algorithm obtains relatively low efficiency about the graphics layout and is not applicable in practical engineering. The critical polygon algorithm as a direct arranging algorithm can get better material utilization, but not be suitable to concave parts, and requires a large and complex time and space [15]. 
In this paper, the storage tank bottom arranging and patchwork problem as shown in Fig. (1), is researched deeply. The annular edge plates are not needed when the storage tank inner diameter is smaller than $12.5 \mathrm{~m}$, while the diameter is greater than or equal to $12.5 \mathrm{~m}$, the annular edge plates are needed. The strip center plates are used and arranged in horizontal direction from the center point of the first quadrant, both central symmetry mode and the center span symmetry mode can be chosen, the plates are arranged with crisscross type and up to the top, and one or two plate width is left on the top. Fig. (1a and b) show the center span symmetry mode; on the basis of the arranging theories, the patchwork theories in this paper are researched deeply. The arranging and patchwork system is designed and developed with the heuristic packing algorithm with the graphics position strategy and with dynamic decision layout order, basing on Sinovation platform, using the object-oriented programming language $\mathrm{C}++$. And the system application researches are also performed.

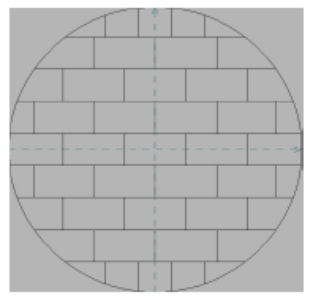

(a) The tank bottom structure with the inner diameter less than $12.5 \mathrm{~m}$

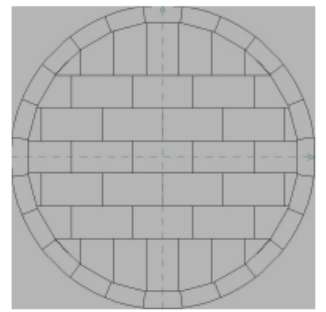

(b) The tank bottom structure with the inner diameter bigger than $12.5 \mathrm{~m}$

Fig. (1). The storage tank bottom structure.

\section{THEORETICAL RESEARCHES ON PATCHWORK PROBLEM OF THE STORAGE TANK BOTTOM}

The patchwork problem of the storage tank bottom belongs to special-shaped parts packing problem, consists of three modules of drawing pretreatment, drawing position strategy and drawing packing order.

\subsection{Drawing Pretreatment}

In this paper, it is necessary to consider the material utilization ratio in drawing pretreatment. The separate plate drawings after arranging have various shapes, and some drawings with curved segments are difficult to patch together. Up to now, there have been little mature theories to patch the curved segments together directly, so it is necessary to discretize arcs into straight lines infinitely at first.

During the discrete process, both the complexity of drawing position strategy and the position accuracy within the control range are necessary to consider. The separate and standard plates are handled as drawings. The separate plates with circular arcs will process by rule. All drawings are eventually converted into triangles, rectangles or trapezoids. Parts of the simplified drawings are shown in Fig. (2).

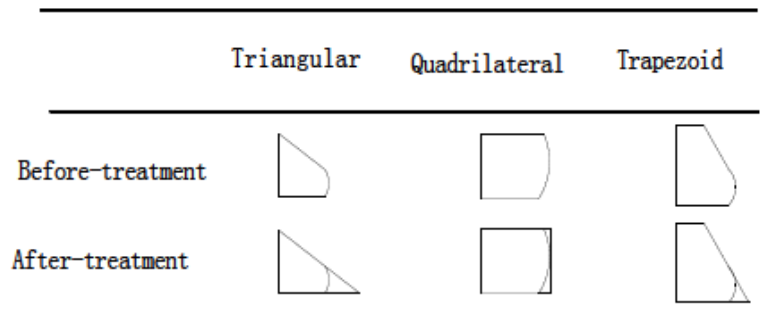

Fig. (2). The pretreatment drawings.

\subsection{Drawing Position Strategy}

Some constraints are needed to be imposed on the piece position, in order to reduce the searching space of the packing program, to exclude packing programs with piece overlaps, which makes sure that each packing program satisfies the principle of non-overlapping with other pieces. The constraints are known as piece position strategy $[15,16]$. The piece packing position can be uniquely determined by the position strategy.

According to the features of the separate plates and edge plates, using graphics position strategy with the graphics knowledge, the graphics position strategy involves a large number of translation transformations, rotation transformations and intersection judgments.

\subsubsection{Translation Transformation}

Taking the Cartesian coordinate xoy and Vector $\overrightarrow{O B}$, the coordinate of the point $\mathrm{B}$ is $(\mathrm{a}, \mathrm{b})$, and shifting any point $\mathrm{P}$ $(x, y)$ on the plane to the point $\mathrm{P}^{\prime}\left(x^{\prime}, y^{\prime}\right)$ along Vector $\overrightarrow{O B}$, and let $\overrightarrow{P P^{\prime}}=\overrightarrow{O B}$. Then

$$
\left\{\begin{array}{l}
x^{\prime}=x+a \\
y^{\prime}=y+b
\end{array}\right.
$$

\subsubsection{Rotation Transformation}

If a point $\mathrm{P}\left(x_{1}, y_{1}\right)$ is rotated to another point $\left(x_{2}, y_{2}\right)$, and the corresponding angle is rotated from $\theta_{1}$ to $\theta_{1}+\theta_{2}$, then,

$$
\begin{aligned}
& x_{2}=x_{1} \cdot \cos \theta_{2}-y_{1} \cdot \sin \theta_{2} \\
& y_{2}=x_{1} \cdot \sin \theta_{2}+y_{1} \cdot \cos \theta_{2}
\end{aligned}
$$

\subsubsection{Intersection Judgment}

The tolerance is necessary due to the floating-point errors during the process of the intersection calculation with the computer. It is considered to be a coincidence point when two point coordinates are sufficiently close. Intuitively, the point can be seen as a ball with radius $\varepsilon$, the edge can be seen as the tube with radius $\varepsilon$, and the surface can be seen as the sheet with thickness $2 \varepsilon$. In this paper, the tolerance $\varepsilon$ is $10^{-6}$. The intersection calculations of dot with dot, dot with line, and dot with plane are used in the arranging and patchwork program. 
(a) In order to judge two points in space to be intersecting or coincidence with each other, it is just to be judged whether the distance between two points is less than $\varepsilon$.

(b) Intersection calculation between the point and the line segment.

To the point $\mathrm{P}(x, y, z)$ and the line segment with two endpoints of $\mathrm{P}_{1}\left(x_{1}, y_{1}, z_{1}\right)$ and $\mathrm{P}_{2}\left(x_{2}, y_{2}, z_{2}\right)$, let the distance between point $\mathrm{P}$ and the line segment $\mathrm{P}_{1} \mathrm{P}_{2}$ is $\mathrm{d}$, then,

$$
\begin{aligned}
& d^{2}=\left(x-x_{1}\right)^{2}+\left(y-y_{1}\right)^{2}+\left(z-z_{1}\right)^{2}+ \\
& {\left[\left(x_{2}-x_{1}\right)\left(x-x_{1}\right)+\left(y_{2}-y_{1}\right)\left(y-y_{1}\right)+\right.} \\
& \left.\left(z_{2}-z_{1}\right)\left(z-z_{1}\right)\right]^{2} /\left[\left(x_{2}-x_{1}\right)\left(y_{2}-y_{1}\right)\right. \\
& \left.+\left(z_{2}-z_{1}\right)\right]^{2}
\end{aligned}
$$

When $\mathrm{d}^{2}<\varepsilon^{2}$, the point $\mathrm{P}$ is in the line segment (or its extended line), and whether point $\mathrm{P}$ falls within the effective range of the line segment is needed to make further judgments. Assuming that $\mathrm{x}$ components of the two line segment endpoints are unequal (otherwise, all components are equal, then two endpoints coincidence with each other and degenerate into a point), as long as comparing the coordinate component, when $x-x_{1}$ and $x-x_{2}$ have the opposite symbols it can be concluded that dot $\mathrm{P}$ is in an effective interval of the line segment.

(c) The intersection calculation between the point and the plane.

There are many algorithms used to judge whether a point in a plane is contained in the polygon in the same plane, the cross-product judgment algorithm is introduced in the paper. Assuming $\mathrm{P}_{0}$ is a judgment point, the polygon vertices are arranged in sequence $P_{1} P_{2} \ldots P_{n}$, as shown in Fig. (3). Let $\mathrm{V}_{\mathrm{i}}=\mathrm{P}_{\mathrm{i}}-\mathrm{P}_{0}, \mathrm{i}=1,2, \ldots, \mathrm{n}, \mathrm{V}_{\mathrm{n}+1}=\mathrm{V}_{1}$.
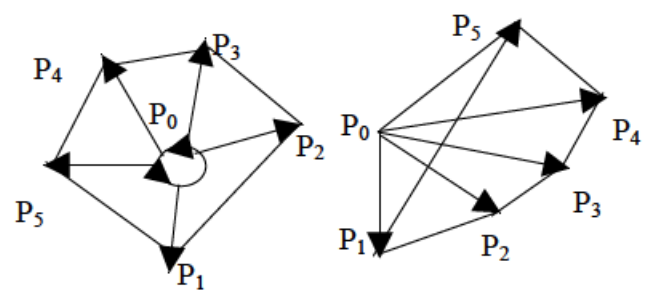

Fig. (3). The polygon with seriated vertices.

The necessary and sufficient condition of the point $\mathrm{P}_{0}$ in the polygon is that the cross products of $\mathrm{V}_{\mathrm{i}} \times \mathrm{V}_{\mathrm{i}+1}(\mathrm{i}=1,2$, $\ldots, n$ ) have the same symbols. The cross-product judgment algorithm is shown in Fig. (4). The cross-product judgment method is only suitable to convex polygon [17].

\subsection{Patchwork Sequence}

After determining the position strategy, the packing program depends on the piece packing sequencing [18]. In this paper, the heuristic algorithm with dynamic decision packing order [15] is used. The heuristic algorithm is to pack the pieces according to the decreasing order of their areas, considering the pieces utilization in all level, adopting a certain fitness evaluation, and packing the high fitness pieces at first. The rectangular pieces are ordered in decreasing order of their areas and composing an initial sequence $\mathrm{P}=\left\{\mathrm{P}_{1}, \mathrm{P}_{2}\right.$,
$\left.\mathrm{P}_{3}, \ldots \ldots, \mathrm{P}_{\mathrm{n}}\right\}$. The following fitness function is adopted in this paper.

$$
F(p)=\text { area } /(h(p) \times w)
$$

Where, the area is the piece area, $h(p)$ is the piece height, $w$ is the piece width.

The following is the process of the heuristic algorithm with dynamic decision packing order:

Step 1 The pieces to be packed are ordered in decreasing order of their areas.

Step 2 Calculating the utilization of each piece.

Step 3 According to the fitness function, finding out a piece that its utilization ratio sum with the first piece is less than 1, then determining whether it is a standard motherboard, if so, put into this piece, otherwise judging the next piece, so the cycle to determine.

Step 4 Reselecting a motherboard, then return to step 3.

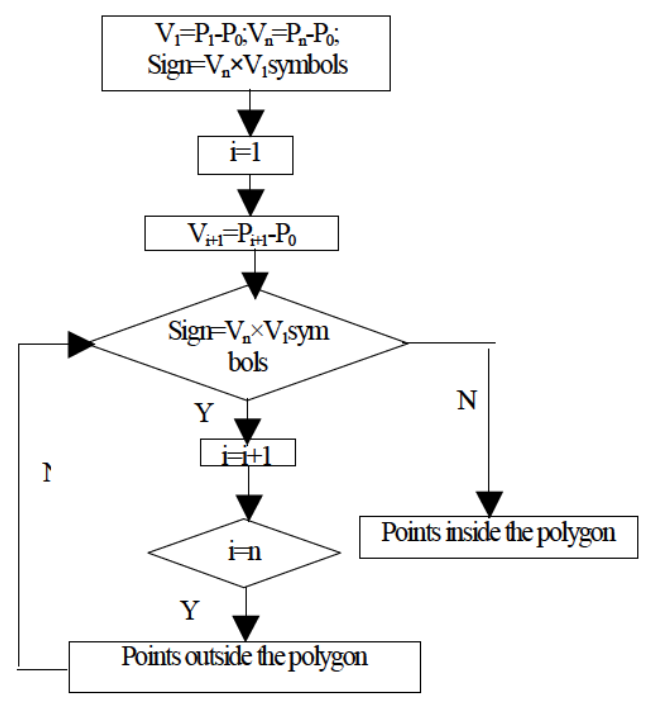

Fig. (4). The cross-product judgment method frame.

\section{DEVELOPMENT OF THE ARRANGING AND PATCHWORK SYSTEM}

\subsection{System Development}

System development is based on the engineering requirements, and using computer algorithms to calculate the research objects. It is necessary to research technical requirements of arranging and patchwork first and design documents according to the specific technical requirements and the theory results, then encode according to design documents.

According to the algorithm studied in the paper, basing on Sinovation platform, using the object-oriented programming language $\mathrm{C}++$ and Visual Studio tools, the arranging and patchwork optimal system is designed and developed. The independent method library is used for the internal calculation of arranging and patchwork program, and the needed results are returned to store. The results are displayed and output graphically with OpenGL. The overall structure of the design is shown in Fig. (5). 


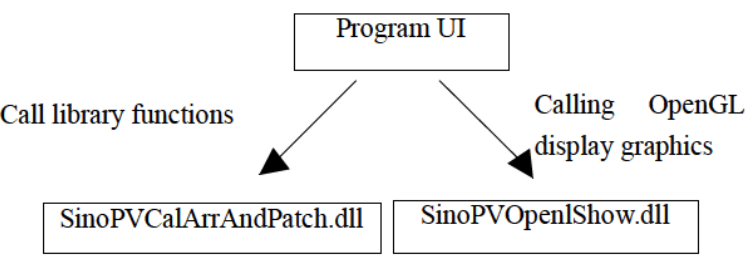

Fig. (5). The overall framework of the system.

\subsection{System Operation Process Flow}

When the program starts, the main interface appears on the screen first, and then users input the required parameters. Arranging and patchwork can be calculated and the engineering drawings can be obtained after entering the correct parameters. The internal program operation flowchart is shown in Fig. (6).

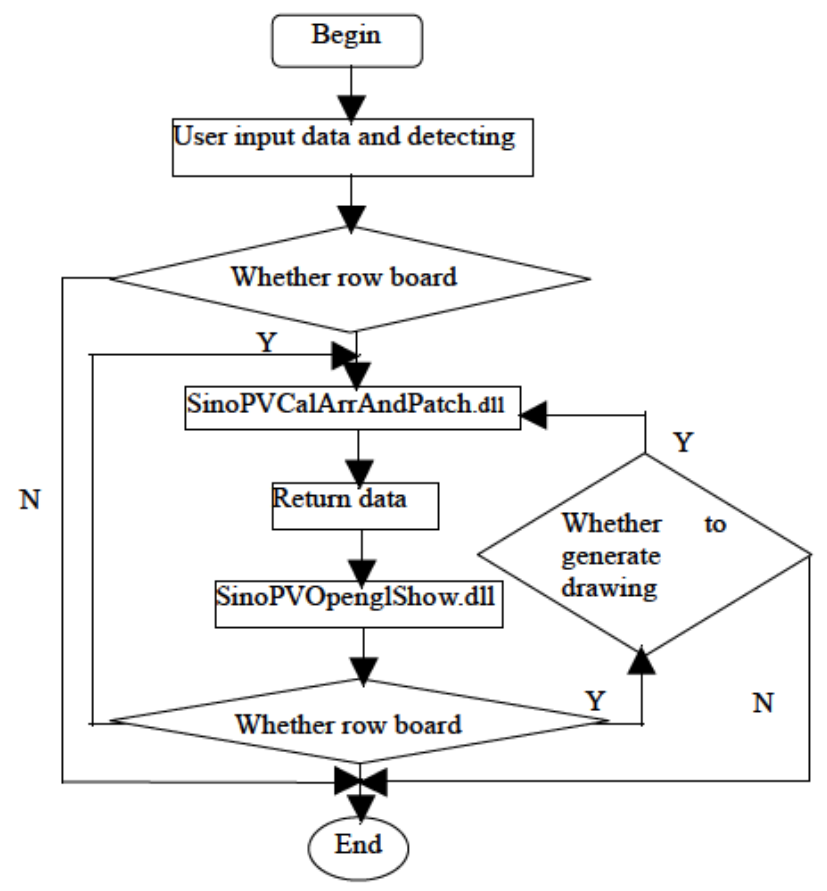

Fig. (6). Internal program execution flowchart.

\section{SYSTEM APPLICATIONS}

\subsection{System Functions and Interface}

The design system has three major function modules.

\subsubsection{Arranging Module}

Users enter the Sinovation interface: by clicking New Drawing command to enter the drawing environment; and by clicking the arranging and patchwork module of the cylindrical storage tank bottom under design structure, to enter into the arranging environment, as shown in Fig. (7).

Users input the relevant parameters based on actual plant requirement, such as the circle bottom inner diameter, the relevant standard plate parameters, and the number of edge plates, select the graphic display modes and arrangement modes, and then click the arranging command button. The interface appears the arranging graphics and shows data results of the arranging statistics, as shown in Fig. (8).

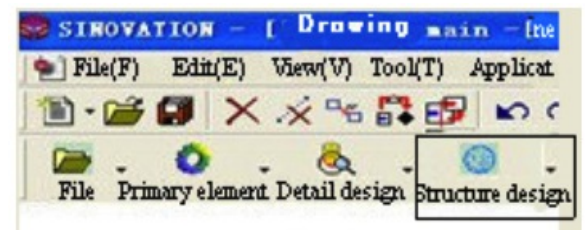

Fig. (7). The icon.

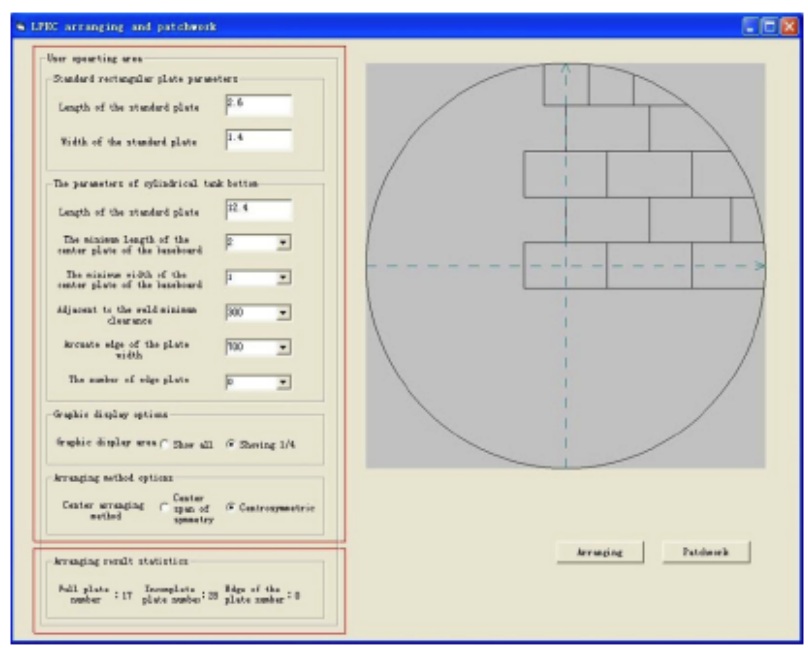

Fig. (8). The arranging interface.

\subsubsection{Patchwork Module}

The main design principles of the patchwork module are to save material and improve the plate utilization ratio. In this module, in order to solve the special-shaped pieces arranging problem, different algorithms are designed and studied. The commonly used one is the rectangular envelopment algorithm [4], which is denoted as algorithm 1 in the paper. The proposed one in the paper is the heuristic packing algorithm with the position strategy based on graphics and with dynamic decision packing order is denoted as algorithm 2 .

Users click patchwork command after arranging and enter into the packing environment under the input parameters, as shown in Fig. (9). In this paper, two kinds of algorithm are designed under patchwork environment and selected by users. The two algorithms may have different advantages and disadvantages, due to different parameters and welding ways. For comparison and judgment, the results will be displayed in patchwork statistical area when users clicking patchwork algorithm command, nesting drawing zone displays graphics and statistical area shows the statistical data. In the drawing area, label 1 represents the actual drawing, label 2 represents packing drawing after regulated processing and label 3 represents standard plate.

\subsubsection{Engineering Drawing Module}

This function module is to unify drawings and data from the arranging and patchwork module to generate the engineering drawing, which makes users easy to save graphics in the form of drawings, and to facilitate factories to cutting stock. In engineering environment, users click on the view command after patchwork, the module will generate engineering drawings with arranging graphics and all the nesting standard plates, as shown in Fig. (10). 


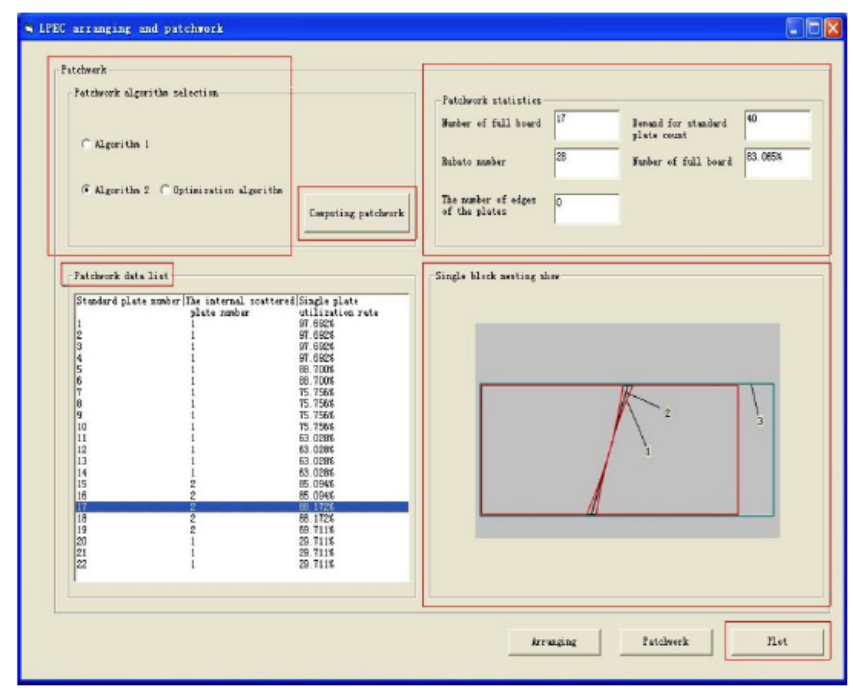

Fig. (9). The patchwork interface.

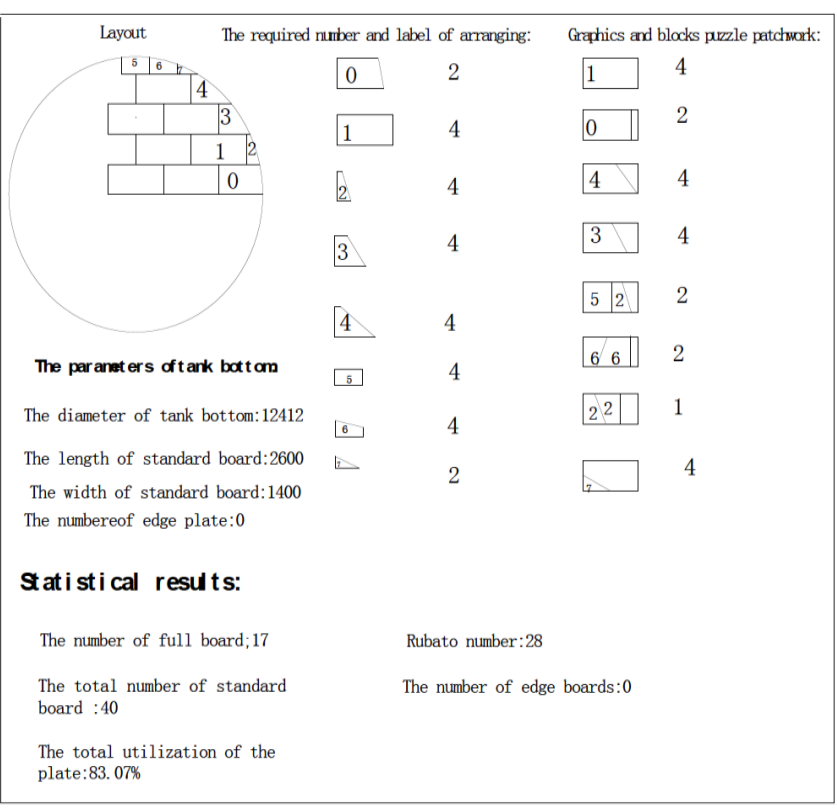

Fig. (10). The engineering drawing.

\subsection{Application Researches and Comparison}

Outputting arranging and patchwork chart after users input the bottom inner diameter, edge plate number, the length and width of the standard plates, and the weld gap, and so on. The example application and the comparison results are as follows:

Under the condition that the standard plate length is $2.6 \mathrm{~m}$ and width is $1.4 \mathrm{~m}$, weld minimum gap is $300 \mathrm{~mm}$, and the other bottom parameters keep constant, when the inner diameter is $12 \mathrm{~m}$, the total number of standard plates is 35 and plates total utilization ratio is $88.906 \%$ according to the algorithm 2. The total number of standard plates is 37 and total utilization ratio of plates is $84.1 \%$ with the algorithm 1 . Comparing with the algorithm 1 , the plate number of algorithm 2 is decreased by $5.4 \%$, plate utilization ratio is increased by $4.806 \%$. When the standard plate inner diameter is 100 , the total number of standard plates is 2262 and the total utilization of plate ratio is $95.531 \%$ with the algorithm 2. The total number of standard plates is 2293 and the total utilization of the plates is $94.239 \%$ with the algorithm 1 . A conclusion can be drawn that the plate number decreases by $1.351 \%$, and the plate utilization ratio increases by $1.292 \%$. Other comparison results can be seen in Table $\mathbf{1}$.

From Table 1, a conclusion can be drawn that the algorithm 2 is superior to algorithm 1 with the same relative parameters. The total number of standard plate is reduced and total plate utilization is increased. Under the researched conditions, the plate total utilization ratio increases by an average of $2.476 \%$. It is validated that the algorithm 2 with graphics position strategy is feasible and superior.

Table 1. The patchwork comparison between algorithm 1 and 2 for different bottom inner diameters and edge plates.

\begin{tabular}{|c|c|c|c|c|}
\hline $\begin{array}{c}\text { Bottom } \\
\text { diameter }(m)\end{array}$ & $\begin{array}{c}\text { The edge } \\
\text { plate (Block) }\end{array}$ & Algorithm & $\begin{array}{l}\text { The stander } \\
\text { plate (Block) }\end{array}$ & $\begin{array}{c}\text { The total } \\
\text { utilization of } \\
\text { the plate }(\%)\end{array}$ \\
\hline \multirow{2}{*}{12} & \multirow{2}{*}{0} & 1 & 37 & 84.100 \\
\hline & & 2 & 35 & 88.906 \\
\hline \multirow{2}{*}{12.4} & \multirow{2}{*}{0} & 1 & 41 & 81.039 \\
\hline & & 2 & 40 & 83.065 \\
\hline \multirow{2}{*}{20} & \multirow{2}{*}{36} & 1 & 125 & 69.149 \\
\hline & & 2 & 117 & 73.877 \\
\hline \multirow{2}{*}{80} & \multirow{2}{*}{120} & 1 & 1499 & 92.260 \\
\hline & & 2 & 1482 & 93.319 \\
\hline \multirow{2}{*}{100} & \multirow{2}{*}{140} & 1 & 2293 & 95.531 \\
\hline & & 2 & 2262 & 94.239 \\
\hline \multirow{2}{*}{120} & \multirow{2}{*}{160} & 1 & 3263 & 95.363 \\
\hline & & 2 & 3231 & 96.308 \\
\hline
\end{tabular}

\section{CONCLUSION}

Basing on the mature arranging theories of strip pieces, theoretical researches on patchwork of storage tank bottom were carried out. According to the heuristic layout algorithm with dynamic decision layout order and based on graphics position strategy proposed in the paper, basing on Sinovation platform, using the object-oriented programming language $\mathrm{C}++$ and Visual Studio tools, the arranging and patchwork system is developed. The system application results show that, compared with the rectangular envelopment algorithm, the plate utilization ratio is developed. The system application results show that, compared with the rectangular envelopment algorithm, the plate utilization ratio is increased with the heuristic layout algorithm.

The developed system is integrated with Sinovation, and easy to operate, and the system also provides a variety of patchwork algorithms for users to select according to different technology requirements. These algorithms have high 
computational efficiency, and users can obtain satisfactory results quickly. With the developed arranging and patchwork system, the labor intensity of the technical engineers is reduced greatly, and their work efficiency is improved.

\section{CONFLICT OF INTEREST}

The authors confirm that this article content has no conflict of interest.

\section{ACKNOWLEDGEMENTS}

This paper is supported by International Cooperation Project from Chinese Ministry of Science and Technology (2011DFB11490), guiding plan project of Chinese Coal Industry Association of Science and Technology (MTKJ2012347 ), and by postgraduate innovation fund project of Shandong University of Science and Technology (YC140206).

\section{REFERENCES}

[1] D. Liu, and J. Song, "The computer patchwork program of steel cylindrical storage tank", Petroleum Engineering Construction, vol. 30, no. 1, pp. 54-56, February 2004.

[2] B. Xu, "The computer arranging technology of cylindrical pressure vessel and vertical storage tank", Petroleum Engineering Construction, vol. 18, no. 6, pp. 44-46, November 1999.

[3] D. Wang, "Comparing the welding critical width between t-shape arranging and long strip arranging of storage tank bottom", Chemical Construction Technology, no. 2, pp. 14-16, 1997.

[4] P. Wei, "Research on leather optimal nesting program", $\mathrm{PhD}$ thesis, Huaqiao University, Fujian, China, June 2011.

[5] M.C. Riff, X. Bonnaire, and B. Neveu, "A revision of recent approaches for two-dimensional strip-packing problems", Engineering Applications of Artificial Intelligence, vol. 22, no. 4-5, pp. 823-827, June 2009.

[6] J.A. Bennell, L.S. Lee, and C.N. Potts, "A genetic algorithm for two dimensional bin packing with due dates", International
Journal of Production Economics Computers, vol. 145, no. 2, pp. 547-560, 2013.

[7] B. Li, S. Wang, X. Shi, and J. Hu, "The rectangular optimal nesting problem based on heuristic algorithm with dynamic decompose", Computer Application, vol. 33, no. 7, pp. 1908-1911, July 2013.

[8] L. Zhang, and J. Li, "On Progress of Cutting Stocks Problem and its Algorithm", Manufacturing Automation, vol. 32, no. 5, pp. 9396, March 2010.

[9] B. Li, "Research on two-dimensional irregular parts packing optimization", $\mathrm{PhD}$ thesis, Jilin University, Jilin, China, June, 2012.

[10] A. Bortfeldt, "A Reduction Approach for Solving the Rectangle Packing Area Minimization Problem", European Journal of Operation Research, vol. 224, no. 3, pp. 486-496, February 2013.

[11] J.A. Bennell, and X. Song "A Beam Search Implementation for the Irregular Shape Packing Problem", Heuristics, vol. 16, pp. 167-188, 2010.

[12] M.T. Costa, A.M. Gomes, and J.F. Oliveira, "Heuristic Approaches to Large-scale Periodic Packing of Irregular Shapes on a Rectangular Sheet", European Journal of Operational Research, vol. 192, no.1, pp. 29-40, January 2009.

[13] L. Li, Y. Zhang, and B. Gao, "Development of Plate Arranging and Patchwork Software for the Bottom of Large Cylindrical Storage Tank", Petrochemical Equipment, vol. 37, no. 4, pp. 6972, July 2008.

[14] G. Li, "Research on the theory and methods of layout design and their applications", PhD thesis, Dalian University of Technology, Dalian, China. June, 2003.

[15] H. Liu, "Research of two-dimensional nesting algorithm based on no-fit polygon", $\mathrm{PhD}$ thesis, Shanghai Jiaotong University, Shanghai, China. June, 2007.

[16] F. Zhang, "Research of packing problem of difform parts based on contour feature location algorithm", $\mathrm{PhD}$ thesis, Jilin University, Jilin, China. July, 2011.

[17] Y. Mao, "The development of computer optimal layout system for blank nesting”, $\mathrm{PhD}$ thesis, Dalian Jiaotong University, Dalian, China, June, 2010.

[18] Y. Zhang, and X. Chen, "Optimal Test Points Selection Based on Multi-Objective Genetic Algorithm", Journal of Electronic Science and Technology of China, vol. 7, no. 4, pp. 317-321, December 2009.

Received: December 15, 2014

Revised: January 04, 2015

Accepted: February 25, 2015

(C) Junru et al.; Licensee Bentham Open.

This is an open access article licensed under the terms of the Creative Commons Attribution Non-Commercial License (http://creativecommons.org/licenses/by-nc/4.0/) which permits unrestricted, non-commercial use, distribution and reproduction in any medium, provided the work is properly cited. 